\title{
Optical frequency comb stabilization of a gigahertz semiconductor disk laser
}

Nayara Jornod, Kutan Gürel, Valentin Wittwer, Pierre Brochard, Sargis Hakobyan, et al.

Nayara Jornod, Kutan Gürel, Valentin J. Wittwer, Pierre Brochard, Sargis Hakobyan, Stéphane Schilt, Dominik Waldburger, Ursula Keller, Thomas Südmeyer, "Optical frequency comb stabilization of a gigahertz semiconductor disk laser," Proc. SPIE 10638, Ultrafast Bandgap Photonics III, 106381W (8 May 2018); doi: 10.1117/12.2318621

SPIE. Event: SPIE Defense + Security, 2018, Orlando, FL, United States 


\title{
Optical frequency comb stabilization of a gigahertz semiconductor disk laser
}

\author{
Nayara Jornod ${ }^{\mathrm{a}}$, Kutan Gürel ${ }^{\mathrm{a}}$, Valentin J. Wittwer ${ }^{\mathrm{a}}$, Pierre Brochard ${ }^{\mathrm{a}}$, Sargis Hakobyan ${ }^{\mathrm{a}}$, \\ Stéphane Schilt ${ }^{\mathrm{a}}$, Dominik Waldburger ${ }^{\mathrm{b}}$, Ursula Keller ${ }^{\mathrm{b}}$, and Thomas Südmeyer*a \\ ${ }^{a}$ Laboratoire Temps-Fréquence, Université de Neuchâtel, CH-2000 Neuchâtel, Switzerland \\ ${ }^{b}$ Department of Physics, Institute for Quantum Electronics, ETH Zürich, CH-8093 Zürich, \\ Switzerland
}

\begin{abstract}
Semiconductor lasers are a promising technology to make optical comb systems more accessible and cost-efficient. We stabilized the carrier-envelope offset (CEO) frequency of a semiconductor disk laser. The laser was modelocked by a SESAM and generates pulses at a wavelength of $1034 \mathrm{~nm}$. It operates at a repetition frequency of $1.8 \mathrm{GHz}$. The 270 -fs pulses are amplified to $3 \mathrm{~W}$ and compressed to $120 \mathrm{fs}$. A coherent octave-spanning supercontinuum spectrum is generated in a highly nonlinear fiber. Using a standard $f$-to- $2 f$ interferometer, we detect the CEO beat with a signal-tonoise ratio of $\sim 30 \mathrm{~dB}$. By applying a feedback signal to the pump current, the CEO frequency is phase-locked to an external reference.
\end{abstract}

Keywords: Frequency combs, ultrafast lasers, VECSELs, semiconductor disk lasers, noise characterization, gigahertz repetition rate lasers, femtosecond lasers

\section{INTRODUCTION}

Optical frequency combs ${ }^{1-3}$ based on ultrafast lasers are used in multiple applications such as frequency metrology or spectroscopy. The vertical external-cavity surface-emitting laser (VECSEL) ${ }^{4}$ technology, also called semiconductor disk lasers (SDLs), offers a high integration factor and the potential for low-cost and high volume production thanks to the wafer-scale manufacturing of the semiconductor gain material and saturable absorber. However, it has been an open question for a long time whether the carrier-envelope offset (CEO) frequency stabilization of an ultrafast VECSEL was possible due to the high nonlinearities and the need for strongly multi-transverse-mode pumping scheme of VECSELs.

Modelocking of VECSELs can easily be achieved with a semiconductor saturable absorber mirror (SESAM) ${ }^{5}$ leading to a simple and compact cavity with high repetition rates up to tens of $\mathrm{GHz}^{6-10}$. VECSELs have demonstrated high output powers in the ps- to fs-pulse duration regime, have excellent amplitude noise properties and achieve low timing jitter ${ }^{11}$. Even more compact pulsed laser sources can be obtained with the modelocked integrated external-cavity surfaceemitting laser (MIXSEL) configuration ${ }^{12}$, where the saturable absorber and the gain medium are integrated into a single semiconductor chip.

In 2014, the first CEO beat from a VECSEL was detected after external amplification and pulse compress ${ }^{13}$. However, the signal-to-noise ratio (SNR) of the obtained CEO beat was too low to enable a detailed noise characterization or its frequency stabilization. In 2016, we presented an indirect method to characterize the frequency noise of the CEO signal of a VECSEL, as well as the response of the CEO frequency $f_{\text {CEO }}$ to a modulation of the pump power, obtained without directly detecting the CEO beat by a self-referencing method, but using an auxiliary continuous-wave laser ${ }^{14}$. These results opened the door to the self-referenced CEO stabilization of a semiconductor disk laser by showing that a feedback bandwidth of a few hundred $\mathrm{kHz}$ should be sufficient and achievable by direct pump current modulation.

In this work, we present a direct noise characterization and the frequency stabilization of the CEO beat of a modelocked SDL. The laser is a 1.8-GHz SESAM-modelocked VECSEL emitting pulses of $270 \mathrm{fs}$ at $1034 \mathrm{~nm}$. Its peak power was insufficient for the direct generation in a photonic crystal fiber (PCF) of the coherent octave-spanning supercontinuum (SC) spectrum that is required for the $f$-to- $2 f$ self-referencing scheme. Therefore, we first amplified the laser pulses in a $3.5-\mathrm{m}$ long polarization-maintaining double-clad $\mathrm{Yb}$-fiber amplifier. The $6-\mu \mathrm{m}$ core diameter enabled spectral broadening in the amplifier, resulting in $6 \mathrm{~W}$ of average power with more than $25 \mathrm{~nm}$ of full width at half maximum (FWHM) optical bandwidth. The pulses were then compressed through transmission gratings to sub-120 $\mathrm{fs}$ at $3-\mathrm{W}$

Ultrafast Bandgap Photonics III, edited by Michael K. Rafailov, Proc. of SPIE Vol. 10638,

$106381 \mathrm{~W} \cdot$ (C) $2018 \mathrm{SPIE} \cdot$ CCC code: 0277-786X/18/\$18 · doi: 10.1117/12.2318621 
average power, from which $1.65 \mathrm{~W}$ were coupled into a $1-\mathrm{m}$ long PCF for the SC generation. A CEO beat with $\sim 30 \mathrm{~dB}$ SNR (in a resolution bandwidth of $300 \mathrm{kHz}$ ) was detected in a quasi-common path $f$-to- $2 f$ interferometer and phaselocked to an external frequency reference with a feedback applied to the current of the VECSEL pump diode, demonstrating efficient noise reduction at low Fourier frequencies. We have thereby confirmed the suitability of ultrafast VECSELs for the generation of stable frequency combs.

The setup and the experimental results presented in this article were described in details in our recent journal publication $^{15}$.

\section{EXPERIMENTAL SETUP}

\subsection{Ultrafast SDL}

The 1.8-GHz semiconductor disk laser is a SESAM-modelocked VECSEL prototype designed at ETH Zürich. The laser cavity consists of only three elements mounted in a V-shaped configuration, the VECSEL gain chip as folding mirror, a SESAM for modelocking operation at one cavity end and an output coupler (radius of curvature of $100 \mathrm{~mm}$, transmission of $1.0 \%$ ) at the other end. The VECSEL and SESAM structures are similar to the one described in Ref ${ }^{8}$. The cavity is housed in an aluminum box to sustain high stability and low noise operation. The temperature of the SESAM and of the VECSEL chip is regulated with thermo-electrical coolers to $21^{\circ} \mathrm{C}$ and $19^{\circ} \mathrm{C}$, respectively, for more stable operation.

The VECSEL gain chip is optically-pumped by a volume Bragg grating (VBG) wavelength-stabilized fiber-coupled laser diode (LIMO35-F100-DL808-EX2009) emitting at $808 \mathrm{~nm}$ with a typical pump power of $9 \mathrm{~W}$. In parallel to the high constant current source (Delta Elektronika SM 7.5-80), the pump diode is driven by a home-built voltage-to-current converter that provides a high-bandwidth modulation channel of the pump power for CEO frequency stabilization. In addition, a low-pass RC filter with a cut-off frequency of $\sim 16 \mathrm{~Hz}$ is placed between the current source and the pump diode to filter out the driver noise and furthermore prevents any cross-talk between the two current drivers.
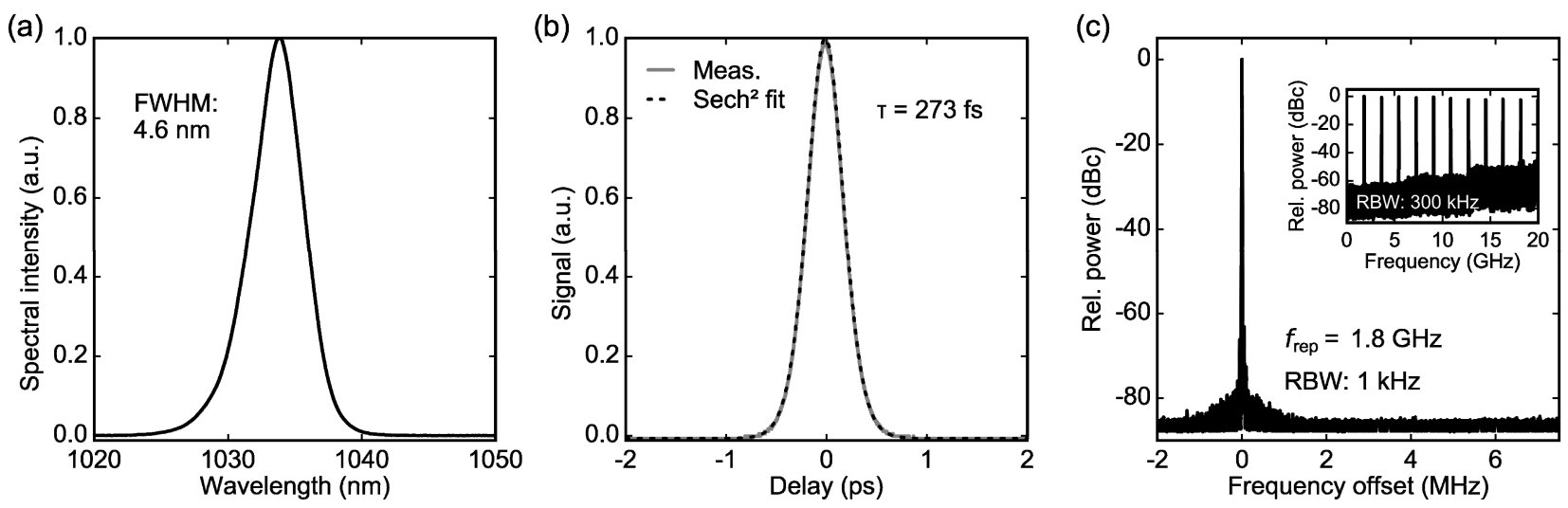

Figure 1. (a) Normalized optical spectrum of the VECSEL with a central wavelength of $1033.9 \mathrm{~nm}$ and a full width at half maximum (FWHM) of $4.6 \mathrm{~nm}$. (b) Intensity autocorrelation trace (grey curve) and corresponding sech ${ }^{2}$ fit (dashed black curve). (c) Microwave spectrum of the VECSEL with a repetition frequency of $1.8 \mathrm{GHz}$ (1-kHz resolution bandwidth, RBW). Inset: Large span microwave spectrum at a RBW of $300 \mathrm{kHz}$.

The laser emits pulses with a duration of $273 \mathrm{fs}$ at a wavelength of $1033.9 \mathrm{~nm}$ with a FWHM spectral bandwidth of $4.6 \mathrm{~nm}$, close to the transform limit (Figure 1). The average output power is $60 \mathrm{~mW}$, which corresponds to a peak power in the range of $100 \mathrm{~W}$. This peak power is insufficient for the generation of a coherent octave-spanning SC spectrum in a nonlinear fiber. Therefore, we implemented an $\mathrm{Yb}$-fiber amplification stage before the self-referencing CEO frequency detection scheme. 
An overview of the complete setup is depicted in Figure 2. The laser output is protected by an optical isolator to prevent any back-reflection from disturbing the laser operation.

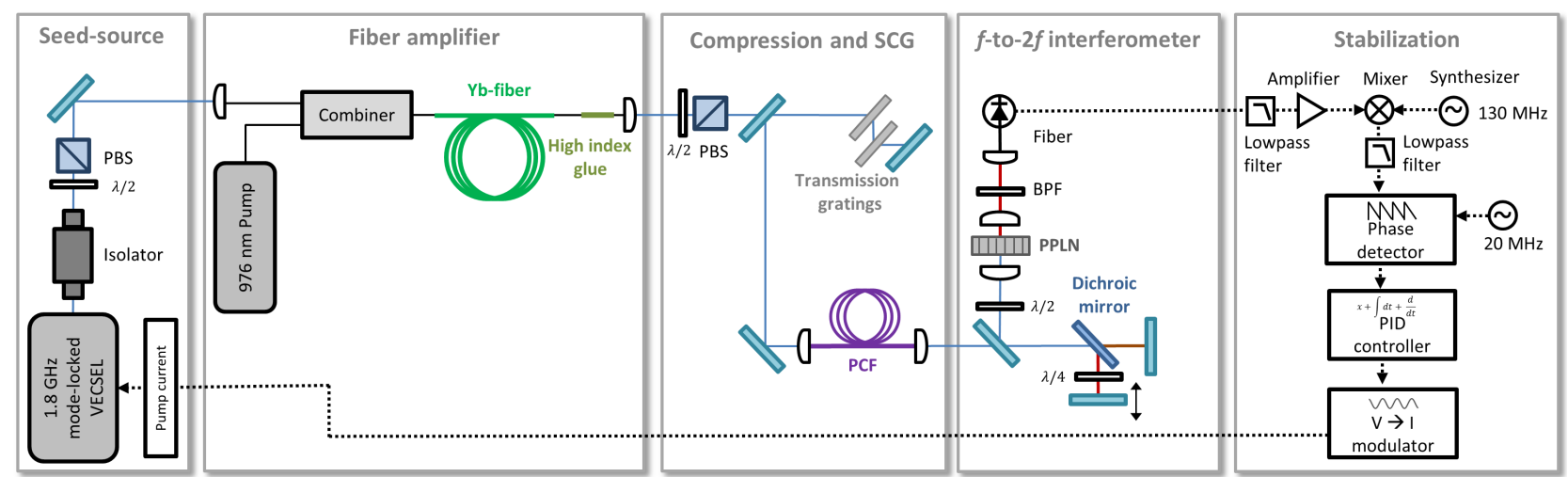

Figure 2. Overall setup of the experiment for the detection and stabilization of the CEO frequency of the VECSEL, comprising the fiber amplification stage, the pulse compression and supercontinuum generation (SCG) stages, the $f$-to- $2 f$ interferometer and the stabilization electronics. PBS: Polarizing beamsplitter; PCF: Photonic crystal fiber; PPLN: Periodically-poled lithium niobate crystal; BPF: Bandpass optical filter; PID: Proportional-integral-derivative controller.

\subsection{Yb-Fiber Amplifier}

The amplifier consists of a segment of $3.5 \mathrm{~m}$ of $\mathrm{Yb}$-doped polarization-maintaining (PM) double-clad fiber with a core diameter of $6 \mu \mathrm{m}$ (Coractive DF-YB-6/128S-PM). A commercial VBG-stabilized fiber-coupled laser diode (Dilas I5F1P15-976.1-25C-HS1.4) emitting at $976 \mathrm{~nm}$ pumps the fiber in the forward direction (see setup in Figure 2). We spliced a multimode pump combiner (MPC) to combine the 976-nm pump and the 1034-nm polarized signal. In addition, a piece of $\sim 40 \mathrm{~cm}$ of passive PM fiber is spliced at the end of the gain fiber, where the unabsorbed pump light is removed from the fiber cladding using high refractive index acrylate coating. This prevents thermal issues at the fiber tip and the use of an additional optical filter at the output.

The laser signal first goes through a half-waveplate and a polarizing beamsplitter to select the desired polarization and is then coupled into a PM fiber with an efficiency of $88 \%$. An average optical power of $46 \mathrm{~mW}$ is seeded in the amplifier.
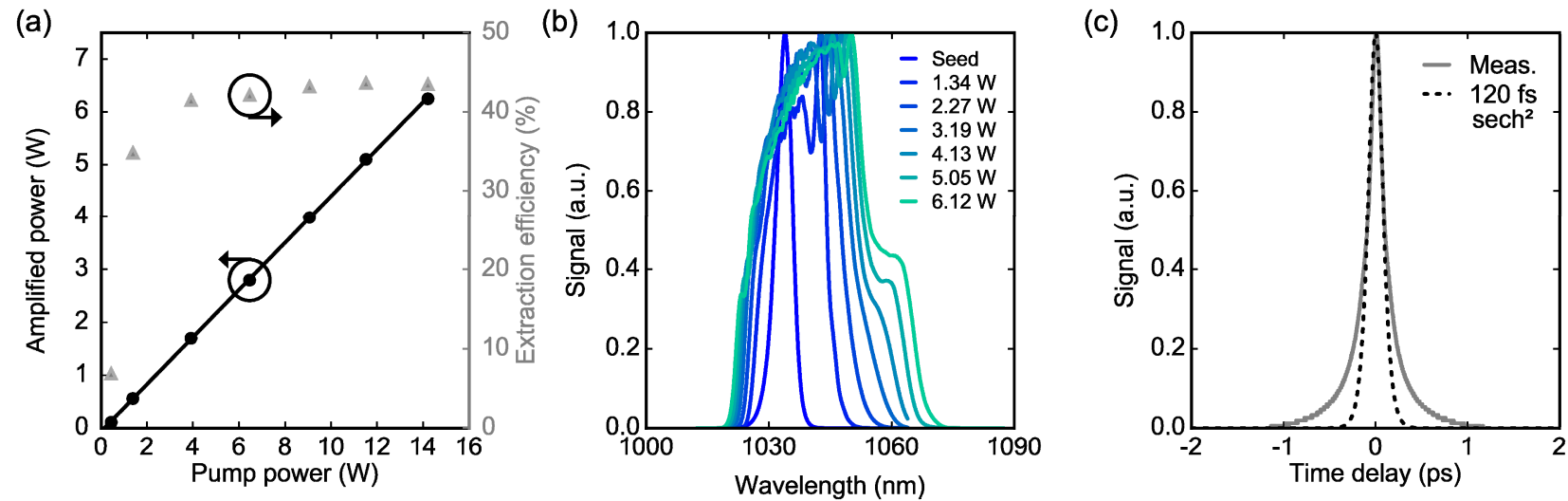

Figure 3. (a) Amplified signal power as a function of the pump power (left axis) and corresponding extraction efficiency (right axis). (b) Normalized optical spectrum of the signal after amplification for various amplified power levels. (c) Intensity autocorrelation trace of the compressed pulses (grey) and comparison with a 120 -fs $\operatorname{sech}^{2}$ pulse (dashed black). 
The pump diode can deliver up to $25 \mathrm{~W}$, from which only $14 \mathrm{~W}$ are used for the typical operation of the amplifier. The seed signal is amplified to more than $6 \mathrm{~W}$ [Figure 3(a)]. The corresponding amplification factor is in the order of $\sim 130$ $(21 \mathrm{~dB})$. The extraction efficiency, computed as the ratio between the amplified output power minus the seed power and the pump power, is $44 \%{ }^{15}$.

The small core diameter of the gain fiber $(6 \mu \mathrm{m})$ together with the additional passive PM fiber segment at the amplifier end enable spectral broadening directly in the amplifier. Figure 3(b) shows the measured amplified optical spectrum for different amplified output powers. The initial 4.6-nm optical bandwidth (FWHM) of the laser is broadened to more than $25 \mathrm{~nm}$ in the amplifier.

During the amplification, the pulse duration is also enlarged to more than 2 ps. After the amplification, the pulses are temporally compressed down to sub-120 fs by two transmission gratings in a double-pass configuration (Figure 2). After amplification and compression, the average power was in the order of $3 \mathrm{~W}$. In the ideal case of perfect $\operatorname{sech}^{2}$ pulses, an optical spectrum with a bandwidth of $25 \mathrm{~nm}$ at a central wavelength of $1034 \mathrm{~nm}$ should be able to support a pulse duration down to $45 \mathrm{fs}$. As seen in Figure 3(c), the autocorrelation trace of the compressed pulses shows small distortions from a sech ${ }^{2}$ pulse shape. We believe that a nonlinear chirp is induced during the amplification process, which explains why the pulses cannot be compressed down to the transform-limited pulse duration supported by the optical bandwidth.

(a)

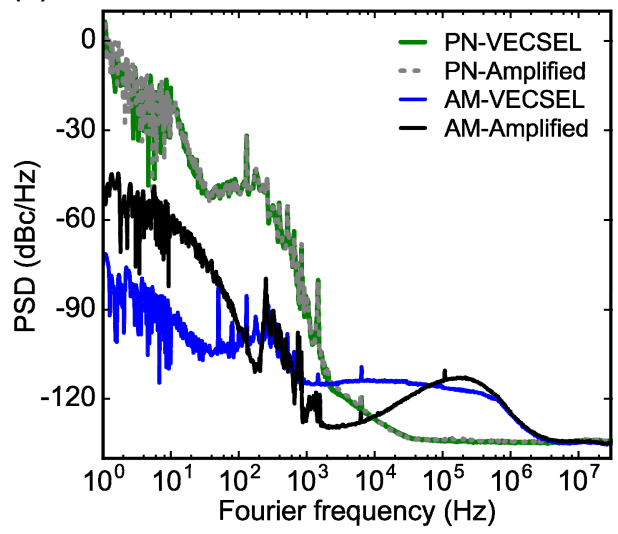

(b)

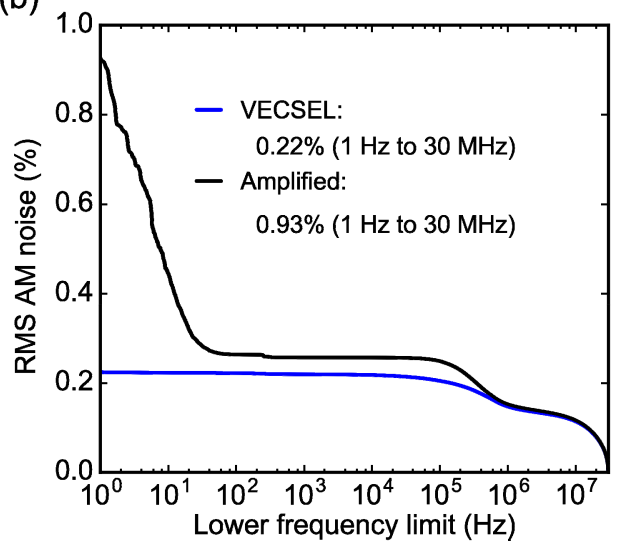

Figure 4. (a) Phase noise (PN) power spectral density (PSD) of the repetition frequency before (green) and after (dashed grey) the amplification, amplitude modulation (AM) noise of the repetition frequency before (blue) and after (black) the amplification. (b) Root mean square (RMS) AM noise as a function of the lower frequency integration limit.

We performed a noise characterization of the ultrafast pulses before and after amplification (at the maximum amplification level) using a phase noise analyzer (Rohde \& Schwarz FSWP26). The phase noise (PN) and amplitude modulation (AM) noise power spectral densities (PSD) are depicted in Figure 4(a). The amplifier adds a negligible phase noise contribution to the signal, and only significant additional AM noise at frequencies below $100 \mathrm{~Hz}$ is observed. The corresponding root mean square (RMS) AM noise increases from $0.2 \%$ to $0.9 \%$ in the range from $1 \mathrm{~Hz}$ to $30 \mathrm{MHz}^{15}$ [Figure 4(b)].

\section{CEO BEAT DETECTION AND STABILIZATION}

After compression, the pulses are seeded into a 1-m long PCF (NKT Photonics NL-3.2-945) for SC spectrum generation. A launched average power of $1.65 \mathrm{~W}$ with a coupling efficiency of $\sim 80 \%$ enables the octave SC generation as expected from simulations [see Figure 5(a)]. The simulated coherence, depicted in Figure 5(c), predicts that the required wavelength for the self-referencing (grey spectral band at $700 \mathrm{~nm}$ and $1400 \mathrm{~nm}$ ) have a high temporal coherence after the SC generation. 
(a)
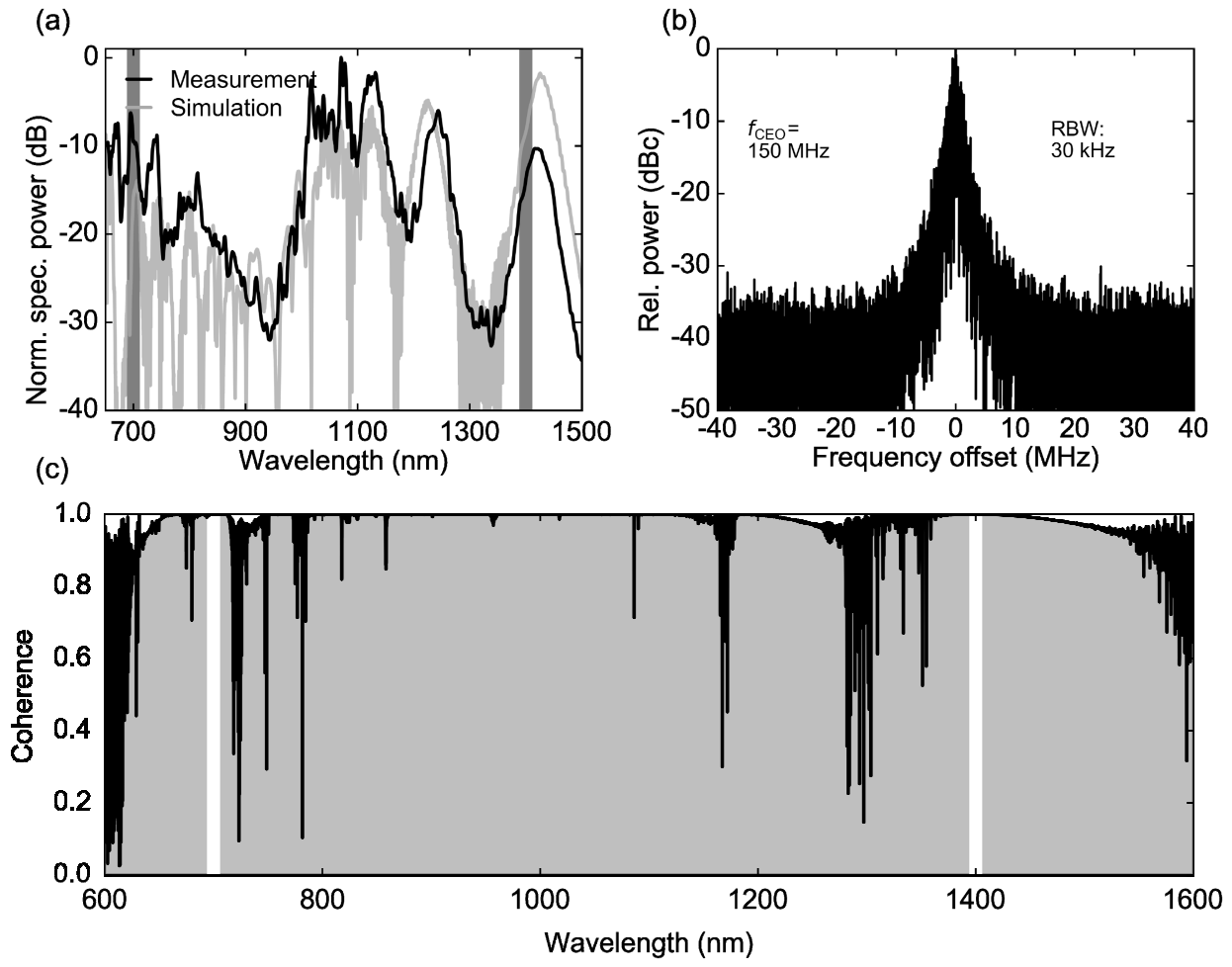

Figure 5. (a) Measured (black) and simulated (grey) octave-spanning SC spectra at the output of the PCF. The grey spectral bands centered at $700 \mathrm{~nm}$ and $1400 \mathrm{~nm}$ are used for CEO beat detection in the $f$-to- $2 f$ interferometer. (b) Detected free-running $\mathrm{CEO}$ beat centered at $150 \mathrm{MHz}(30-\mathrm{kHz} \mathrm{RBW})$. (c) Simulated coherence of the SC spectrum.

The CEO beat note is generated in a quasi-common-path $f$-to- $2 f$ interferometer schematized in the bottom part of Figure 2. The 700-nm and 1400-nm spectral components co-propagate through a MgO:PPLN (magnesium-oxide-doped periodically-poled lithium niobate) crystal of 1-mm length, where the $1400-\mathrm{nm}$ light is frequency-doubled. The polarization of the two beams is matched using a half-wave plate placed in the common path before the crystal and a quarter-wave plate in the 700-nm adjustable delay line.

The 700-nm light from the two beams is optically band-pass filtered and coupled into a single-mode fiber to ensure an optimum spatial overlap. The resulting CEO beat is detected using a variable-gain avalanche photodetector (Thorlabs APD430A/M) with a bandwidth of $400 \mathrm{MHz}$ in the stabilization experiments and using a fast photodetector (New Focus $1014,45-\mathrm{GHz}$ bandwidth) for static frequency tuning curve measurements. The photodiode output signal is band-pass filtered and amplified. A maximum SNR of $\sim 30 \mathrm{~dB}$ in a $300-\mathrm{kHz}$ RBW was measured. Figure 5(b) shows the typical free-running $\mathrm{CEO}$ beat measured at $\sim 150 \mathrm{MHz}$ and used later for stabilization.

The CEO frequency $f_{\mathrm{CEO}}$ was dependent on the operation mode of the VECSEL and changes in the laser parameters (for example SESAM or gain chip temperature, pump power and fine cavity alignment) were used to tune the CEO beat note to a desired frequency in the detector bandwidth. Figure 6(a) shows the measured tuning curve of $f_{\text {CEO }}$ as a function of the current of the VECSEL pump diode. The CEO frequency was in the range of $500 \mathrm{MHz}$ in this case, with a tuning coefficient of $\sim 0.3 \mathrm{MHz} / \mathrm{mA}$.

To evaluate the possibility to stabilize the CEO frequency by modulating the pump power, we measured the dynamic response of $f_{\text {CEO }}$ to a modulation of the pump current. This measurement was performed using a frequency discriminator ${ }^{16}$ and a lock-in amplifier (Zurich Instrument HF2LI) while modulating the pump current with our fast current driver. The measured transfer function has a $-3-\mathrm{dB}$ cutoff frequency of $\sim 300 \mathrm{kHz}$ with a corresponding phase shift of approximately $-90^{\circ}$ [see Figure 6(b)]. In addition, we also investigated the response of the pump power to a 
modulation of the driving current [grey curves in Figure 6(b)]. The observed modulation bandwidth is larger than $1 \mathrm{MHz}$, which shows that the control of $f_{\text {CEO }}$ is neither limited by the pump driving electronics, nor by the modulation response of the pump diode itself. It is more likely limited by the cavity dynamics of the laser ${ }^{15}$ (the short upper-state lifetime in the semiconductor gain is not expected to be a limitation).
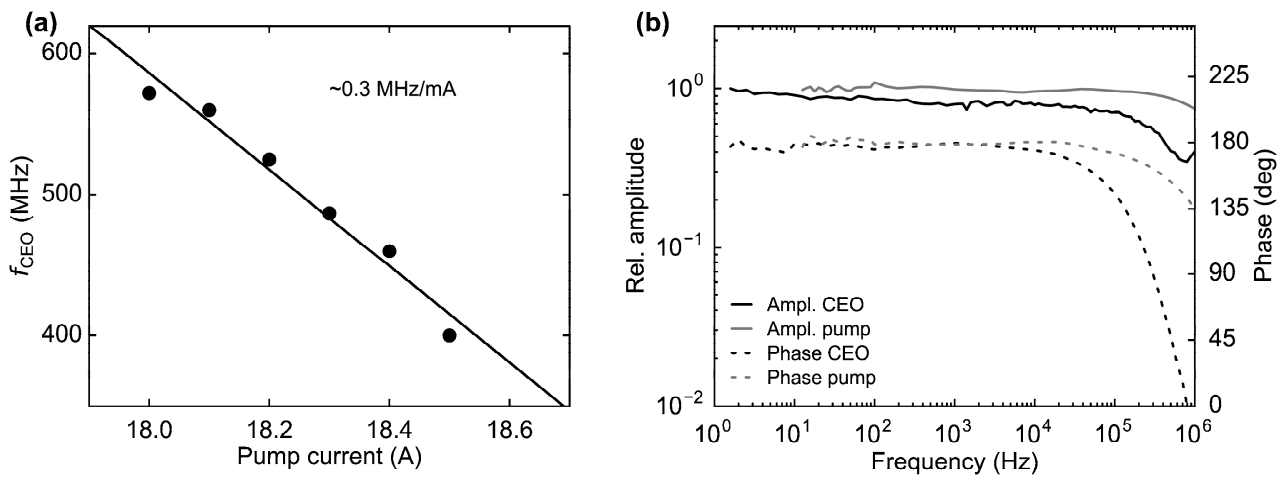

Figure 6. (a) Static tuning curve of the CEO frequency with the pump current. (b) Relative amplitude (left axis) of the transfer functions of the pump optical power (solid grey line) and of $f_{\text {CEO }}$ (solid black line) for a modulation of the pump current. Phase (right axis) of the transfer functions of the pump optical power (dashed grey line) and of $f_{\text {CEO }}$ (dashed black line) for a modulation of the pump current.

The noise characterization of the free-running CEO frequency performed using the same phase noise analyzer as for the amplifier is presented in Figure 7 (black curve). The crossing point of the frequency noise PSD and the $\beta$-separation line provides a good estimation of the bandwidth necessary to achieve a tight lock of the CEO frequency in a feedback loop ${ }^{17}$. The expected feedback bandwidth is in the range of $600 \mathrm{kHz}$, which is nearly two times higher than expected from a previous estimation ${ }^{14}$. The higher required bandwidth is due to the plateau observed at Fourier frequencies above $\sim 1 \mathrm{kHz}$ in the frequency noise PSD. To investigate the origin of this noise, we measured the relative intensity noise (RIN) of the pump diode and calculated its contribution to the CEO frequency noise PSD using the previously recorded frequency response of $f_{\mathrm{CEO}}$ for pump current modulation [Figure 6(b)]. The resulting frequency noise is presented in grey in Figure 7. It overlaps fairly well with the measured free-running CEO frequency noise above $1 \mathrm{kHz}$, revealing that the high $f_{\text {CEO }}$ noise comes from the AM noise of the pump diode ${ }^{15}$.

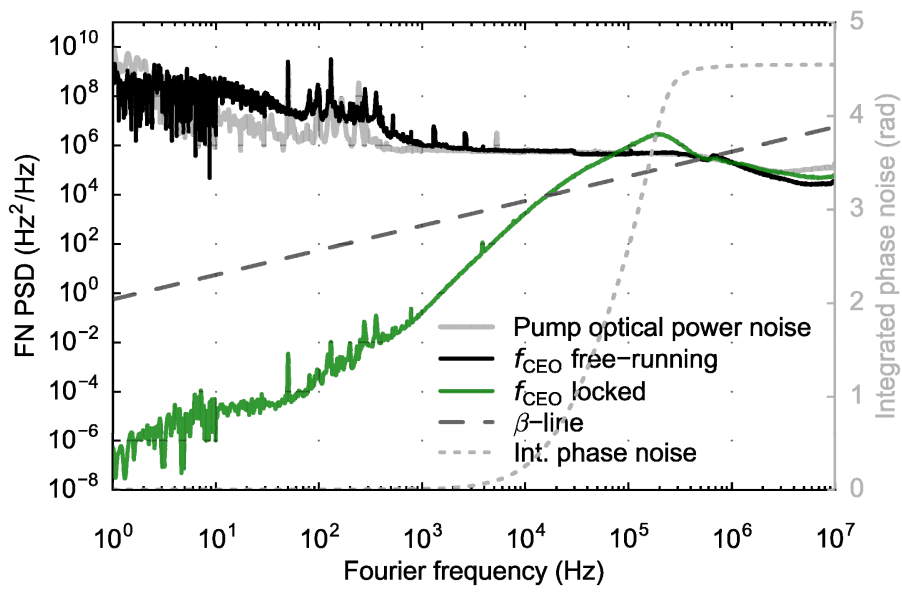

Figure 7. Frequency noise (FN) PSD of the free-running (black) and stabilized (green) CEO signal, and pump-induced frequency noise (grey) calculated from the measured pump RIN multiplied by the pump-power-to- $f_{\text {CEO }}$ transfer function. Right axis: Integrated phase noise of the stabilized CEO signal as a function of the upper cut-off frequency (dashed grey line). 
The CEO frequency stabilization was implemented by first frequency down-converting the CEO beat to $\sim 20 \mathrm{MHz}$, and then comparing this signal to a reference signal from a function generator in a digital phase detector (Menlo Systems DXD200). The phase error signal was sent to an analog proportional-integral-derivative (PID) servo-controller (Vescent Photonics D2-125). The correction signal directly drove the voltage-to-current converter to modulate the VECSEL pump power.

The frequency noise PSD of the stabilized CEO beat is displayed in green in Figure 7. The stabilization shows a clear noise reduction at Fourier frequencies below $\sim 100 \mathrm{kHz}$. For frequencies below $\sim 20 \mathrm{kHz}$, the frequency noise is reduced below the $\beta$-separation line. However, the noise around the servo bump at $\sim 200-\mathrm{kHz}$ still exceeds the $\beta$-separation line, preventing the achievement of a tight lock in the present conditions. The integrated phase noise is 4.5 rad integrated from $1 \mathrm{~Hz}$ to $10 \mathrm{MHz}$. As previously mentioned the AM noise of the pump diode limits the $f_{\text {CEO }}$ free-running noise at high frequencies and increases the requested feedback bandwidth which impedes the demonstration of a tight lock ${ }^{15}$.

\section{CONCLUSION}

We have described the first CEO frequency stabilization of an ultrafast semiconductor laser ${ }^{15}$. The 1.8-GHz VECSEL was first amplified and spectrally-broadened in a double clad fiber amplifier, enabling 120 -fs pulses after compression with an average power of $3 \mathrm{~W}$, resulting in the generation of a coherent octave-spanning supercontinuum spectrum. The CEO frequency was detected in an $f$-to- $2 f$ interferometer with an SNR of $\sim 30 \mathrm{~dB}$ (300-kHz RBW), sufficient for phase stabilization.

The high frequency noise of the free-running CEO beat that has prevented so far the achievement of a tight lock originates from the AM noise of the pump diode. A reduction of this noise, which can be achieved for instance with an intensity stabilization loop, would result in a reduction of the CEO noise and in a enable a better lock.

Moreover, the ultrafast VECSEL technology is steadily evolving, and 101-fs pulses with 1-kW peak power have already been demonstrated ${ }^{18}$. The combination with $\mathrm{mm}$-long silicon nitride waveguides for the SCG recently enabled direct CEO frequency detection of a VECSEL without previous amplification ${ }^{19}$.

\section{REFERENCES}

[1] Telle, H. R., Steinmeyer, G., Dunlop, A. E., Stenger, J., Sutter, D. H. \& Keller, U., "Carrier-envelope offset phase control: A novel concept for absolute optical frequency measurement and ultrashort pulse generation," Appl. Phys. B 69, 327-332 (1999).

[2] Jones, D. J., Diddams, S. A., Ranka, J. K., Stentz, A., Windeler, R. S., Hall, J. L. \& Cundiff, S. T., "CarrierEnvelope Phase Control of Femtosecond Mode-Locked Lasers and Direct Optical Frequency Synthesis," Science 288, 635-639 (2000).

[3] Apolonski, A., Poppe, A., Tempea, G., Spielmann, C., Udem, T., Holzwarth, R., Hänsch, T. W. \& Krausz, F., "Controlling the Phase Evolution of Few-Cycle Light Pulses," Phys. Rev. Lett. 85, 740-743 (2000).

[4] Kuznetsov, M., Hakimi, F., Sprague, R. \& Mooradian, A., "High-Power (>0.5-W CW) Diode-Pumped VerticalExternal-Cavity Surface-Emitting Semiconductor Lasers with Circular TEM $0_{00}$ Beams," IEEE Photonics Technol. Lett. 9, 1063-1065 (1997).

[5] Keller, U., Weingarten, K. J., Kärtner, F. X., Kopf, D., Braun, B., Jung, I. D., Fluck, R., Hönninger, C., Matuschek, N. \& Aus der Au, J., "Semiconductor Saturable Absorber Mirrors (SESAM's) for Femtosecond to Nanosecond Pulse Generation in Solid-State Lasers," IEEE J. Sel. Top. Quantum Electron. 2, 435-453 (1996).

[6] Tilma, B. W., Mangold, M., Zaugg, C. A., Link, S. M., Waldburger, D., Klenner, A., Mayer, A. S., Gini, E., Golling, M. \& Keller, U., "Recent advances in ultrafast semiconductor disk lasers," Light Sci. Appl. 4, e310 (2015).

[7] Scheller, M., Wang, T.-L., Kunert, B., Stolz, W., Koch, S. W. \& Moloney, J. V., "Passively modelocked VECSEL emitting 682 fs pulses with $5.1 \mathrm{~W}$ of average output power," Electron. Lett. 48, 588-589 (2012). 
[8] Waldburger, D., Link, S. M., Mangold, M., Alfieri, C. G. E., Gini, E., Golling, M., Tilma, B. W. \& Keller, U., "High-power 100 fs semiconductor disk lasers," Optica 3, 844-852 (2016).

[9] Lorenser, D., Maas, D. J. H. C., Unold, H. J., Bellancourt, A.-R., Rudin, B., Gini, E., Ebling, D. \& Keller, U., "50GHz Passively Mode-Locked Surface-Emitting Semiconductor Laser With 100-mW Average Output Power," IEEE J. Quantum Electron. 42, 838-847 (2006).

[10] Guina, M., Rantamäki, A. \& Härkönen, A., "Optically pumped VECSELs: review of technology and progress," J. Phys. Appl. Phys. 50, 383001 (2017).

[11] Wittwer, V. J., van der Linden, R., Tilma, B. W., Resan, B., Weingarten, K. J., Südmeyer, T. \& Keller, U., "Sub-60fs Timing Jitter of a SESAM Modelocked VECSEL," IEEE Photonics J. 5, 1400107 (2013).

[12] Maas, D. J. H. C., Bellancourt, A.-R., Rudin, B., Golling, M., Unold, H. J., Südmeyer, T. \& Keller, U., "Vertical integration of ultrafast semiconductor lasers," Appl. Phys. B 88, 493-497 (2007).

[13] Zaugg, C. A., Klenner, A., Mangold, M., Mayer, A. S., Link, S. M., Emaury, F., Golling, M., Gini, E., Saraceno, C. J., Tilma, B. W. \& Keller, U., "Gigahertz self-referenceable frequency comb from a semiconductor disk laser," Opt. Express 22, 16445-16455 (2014).

[14] Brochard, P., Jornod, N., Schilt, S., Wittwer, V. J., Hakobyan, S., Waldburger, D., Link, S. M., Alfieri, C. G. E., Golling, M., Devenoges, L., Morel, J., Keller, U. \& Südmeyer, T., "First investigation of the noise and modulation properties of the carrier-envelope offset in a modelocked semiconductor laser," Opt. Lett. 41, 3165-3168 (2016).

[15] Jornod, N., Gürel, K., Wittwer, V. J., Brochard, P., Hakobyan, S., Schilt, S., Waldburger, D., Keller, U. \& Südmeyer, T., "Carrier-envelope offset frequency stabilization of a gigahertz semiconductor disk laser," Optica 4, 1482 (2017).

[16] Schilt, S., Bucalovic, N., Tombez, L., Dolgovskiy, V., Schori, C., Di Domenico, G., Zaffalon, M. \& Thomann, P., "Frequency discriminators for the characterization of narrow-spectrum heterodyne beat signals: Application to the measurement of a sub-hertz carrier-envelope-offset beat in an optical frequency comb," Rev. Sci. Instrum. 82, $123116(2011)$.

[17] Di Domenico, G., Schilt, S. \& Thomann, P., "Simple approach to the relation between laser frequency noise and laser line shape," Appl. Opt. 49, 4801-4807 (2010).

[18] Waldburger, D., Alfieri, C. G. E., Link, S. M., Gini, E., Golling, M. \& Keller, U., "High-Power Semiconductor Disk Lasers with Record-Short Pulse Durations," in European Conference on Lasers and Electro-Optics - European Quantum Electronics Conference (Optical Society of America, 2017), p. CB-8.1.

[19] Waldburger, D., Mayer, A. S., Alfieri, C. G., Johnson, A. R., Ji, X., Klenner, A., Okawachi, Y., Lipson, M., Gaeta, A. L. \& Keller, U., "Self-referenced CEO Frequency Detection of a Semiconductor Disk Laser using a Silicon Nitride Waveguide," in Advanced Solid State Lasers (Optical Society of America, 2017), p. ATu6A-3. 